\title{
Room-Temperature Synthesis of Ni Nanoparticles as the Absorbent Used for Sewage Treatment
}

\author{
Genhua Zhang, Jianchen Li, Guangshu Zhang, and Lijun Zhao \\ Key Laboratory of Automobile Materials (Jilin University), Ministry of Education and School of Materials Science and Engineering, \\ Jilin University, Changchun 130022, China
}

Correspondence should be addressed to Lijun Zhao; lijunzhao@jlu.edu.cn

Received 18 November 2014; Revised 14 July 2015; Accepted 15 July 2015

Academic Editor: Belal F. Yousif

Copyright (c) 2015 Genhua Zhang et al. This is an open access article distributed under the Creative Commons Attribution License, which permits unrestricted use, distribution, and reproduction in any medium, provided the original work is properly cited.

\begin{abstract}
The magnetic Ni nanoparticles of $10-30 \mathrm{~nm}$ in size were synthesized by the reduction of $\mathrm{Ni}^{2+}$ by $\mathrm{NaBH}_{4}$ at room temperature. The amount of added water in the formation of Ni nanoparticles is a significant factor, which ensures that Ni nanoparticles are not oxidized by oxygen. XRD patterns and FESEM micrographs showed the constituent and structure and micromorphology. Congo red was used as adsorbate to quantitatively examine the adsorption capability of $\mathrm{Ni}$ nanoparticles for the organic dyes in industry wastewater. The magnetic hysteresis measurement indicated that the Ni nanoparticles presented ferromagnetic properties. The experimental results showed the as-obtained Ni nanoparticles might be a potential adsorbent in sewage treatment process.
\end{abstract}

\section{Introduction}

With the development of modern industry, the treatment of various harmful organic chemicals, especially the dye from textiles and mining industries which can affect aquatic life and food chains due to their carcinogenic and mutagenic effects [1], is more and more concerned all over the world. On the other hand, since the end of the 1980s, there had been growing interest in fabrication of nanostructures with specific morphologies and functions. This was due to their significantly different physical, chemical, thermodynamic, and mechanical properties from the bulk counterparts as a result of surface or quantum size effects. Among them, the nanoscaled magnetic materials were paid particular attention for their unique properties and uses in high-density magnetic recording, magnetic sensors, and researching magnetic phenomena in low-dimensional systems [2-6].

In the past decades, magnetic materials are regarded as the potential adsorbents for dyes in sewage owing to their high specific surface area and convenient magnetic separation. Nickel is one of the typical ferromagnetic materials which have been studied for many years [7-12]. Various methods were developed to fabricate Ni nanostructures, such as template synthesis [8], solid-phase pyrolysis of organometallic precursor [13], laser ablation [14], reduction of metal salts $[6,15]$, and solvothermal methods [16]. In solutionphase methods, surfactants were used to prevent the fabricated materials from aggregation. However, because the adsorption process occurs on the metal surfaces, the presence of surfactants goes against the adsorptive applications. Herein, we fabricated Ni nanoparticles free of any surfactants at room temperature in the air, which reduced fabrication cost and contamination to environments compared to methods above, and conformed to the development tendency of green chemistry.

Most often, the aqueous solution of Congo red (CR), a kind of azo dye, was chosen as the sewage to investigate the adsorption capacity of fabricated materials. For instance, as far back as 2002, Namasivayam and Kavitha reported the adsorption capacity of activated carbon prepared from coir pith, an agricultural solid waste, Dyes Pigments, although the equilibrium adsorbed concentration was $6.7 \mathrm{mg} \cdot \mathrm{g}^{-1}$ [17]. In 2010, Afkhami and Moosavi prepared maghemite nanoparticles to remove the Congo red in the aqueous solutions with the equilibrium adsorbed concentration which reached $208.3 \mathrm{mg} \cdot \mathrm{g}^{-1}$ [18]. In our previous works, the removal capacity for Congo red using various micronanomaterials ranged 
from 170 to $800 \mathrm{mg} \cdot \mathrm{g}^{-1}$ [19-21], which have refreshed to $966.7 \mathrm{mg} \cdot \mathrm{g}^{-1}$ in the present work.

\section{Experimental}

All chemicals used in this work were of analytical grade without further purification and were purchased from Sinopharm Chemical Reagent Beijing Co., Ltd. In the typical process, $0.2 \mathrm{~g} \mathrm{NiCl}_{2} \cdot 6 \mathrm{H}_{2} \mathrm{O}$ and $0.1 \mathrm{~g} \mathrm{NaBH}_{4}$ were put into mortar and pestled adequately when mixed powders became black. Then $10 \mathrm{~mL}$ of $\mathrm{H}_{2} \mathrm{O}$ was added into mortar, and the hydrogen gas was released immediately. After the reaction was completed, black precipitate was collected with a magnet and washed with deionized water and ethanol several times. The asobtained powders were naturally dried at ambient temperature in the air until they became dry.

During the reaction process, $\mathrm{NaBH}_{4}$ acted as a reducing agent which reduced the $\mathrm{Ni}^{2+}$ and water to $\mathrm{Ni}^{0}$ and $\mathrm{H}_{2}$ via the following reactions, respectively:

$$
\begin{gathered}
4 \mathrm{Ni}^{2+}+\mathrm{BH}_{4}^{-}+2 \mathrm{H}_{2} \mathrm{O} \longrightarrow 4 \mathrm{Ni}+\mathrm{BO}_{2}^{-}+8 \mathrm{H}^{+} \\
\mathrm{BH}_{4}^{-}+2 \mathrm{H}_{2} \mathrm{O} \longrightarrow \mathrm{BO}_{2}^{-}+4 \mathrm{H}_{2}
\end{gathered}
$$

Usually, the stability of the nanoparticles is apt to be affected by their extreme activity toward water and oxygen. So the amount of $\mathrm{H}_{2} \mathrm{O}$ added into the mortar should be as small as possible to ensure that the $\mathrm{Ni}$ nanoparticles were not oxidized by oxygen dissolving in water. Fortunately, generating hydrogen gas could assist in excluding a large proportion of oxygen in the water, which further avoided the oxidization of Ni nanoparticles.

A standard CR solution with an initial concentration of $130 \mathrm{mg} \cdot \mathrm{L}^{-1}$ was firstly prepared. Then, $6 \mathrm{mg}$ of dry nickel powders was added to $50 \mathrm{~mL}$ of the above solution under stirring. After a specified time, the solid and liquid were separated by a magnet and UV-Vis adsorption spectra were measured by an Agilent Cary 50 UV-Vis spectrophotometer to determine the CR concentration in remaining solutions.

The equilibrium adsorbed concentration, $q_{e}$, was calculated according to the following equation [18]:

$$
q_{e}=\frac{\left(C_{0}-C_{e}\right) \times V}{W},
$$

where $C_{0}$ is the initial concentration of $\mathrm{CR}, \mathrm{mg} \cdot \mathrm{L}^{-3} ; C_{e}$ is the equilibrium concentration in solution, $\mathrm{mg} \cdot \mathrm{L}^{-3} ; \mathrm{V}$ is the total volume of the solution, $\mathrm{dm}^{3}$; and $W$ is the dry mass of the $\mathrm{Ni}$ nanoparticles, g. The equilibrium adsorbed concentration indicates the adsorptive capacity of the measured samples, and all the investigations were carried out in triplicate to avoid any discrepancy in experimental results.

The phases were identified by means of X-ray diffraction (XRD) with a Rigaku D/max 2500pc X-ray diffractometer with $\mathrm{Cu} K \alpha$ radiation $(\lambda=1.54156 \AA)$. The morphologies were characterized by a JEOLJSM-6700F fieldemission scanning electron microscopy (FESEM) operated at an acceleration voltage of $11.0 \mathrm{kV}$. Transmission electron microscopy (TEM) patterns were obtained and were carried out on a JEOL $2100 \mathrm{~F}$ with an emission voltage of $200 \mathrm{kV}$.

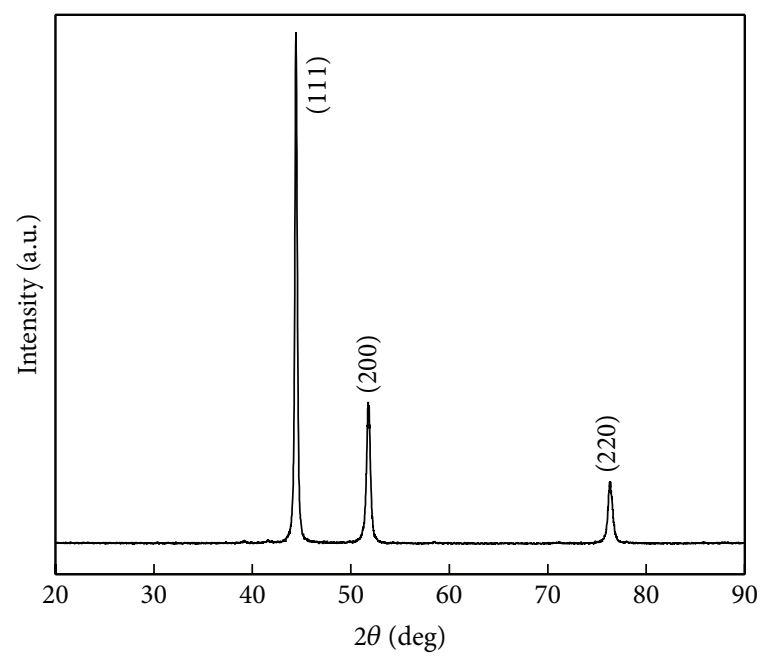

FIGURE 1: XRD pattern of room-temperature prepared Ni nanoparticles.

IR spectra of the samples were characterized using a FTIR spectrophotometer (NEXUS, 670) in KBr pellets. Hysteresis loops of the sample were obtained on a VSM-7300 vibrating sample magnetometer (VSM) at room temperature.

\section{Results and Discussion}

Figure 1 shows the XRD patterns of $\mathrm{Ni}$ nanoparticles. As shown in the patterns, this is typical XRD profile of facecentered-cubic (FCC) nickel phase (JCPDS 04-0850), in which there are three diffraction peaks at $2 \theta=44.5^{\circ}, 51.8^{\circ}$, and $76.4^{\circ}$ corresponding to the crystal planes of (111), (200), and (220).

Figures 2(a) and 2(b) are the FESEM and TEM micrograph, respectively, which show that Ni nanoparticles have irregular shapes with a size range of 10 to $30 \mathrm{~nm}$. This may be because there are no surfactants applied in the reaction process, which made the rapid growth of Ni nanoparticles take place along a prior crystal orientation in the process of violent reaction and finally formed particles of various shapes. On the other hand, small quantity of heat released by the redox reaction only supported the particles to grow up to the size range of $10-30 \mathrm{~nm}$.

Subsequently, the adsorption capacity of Ni powders for CR was investigated. The molecular structure of CR is shown in Figure 3(a). As shown in Figure 3(a), the variation of the adsorption amount with adsorption time, which illustrates that $83 \%$ of CR can be removed after $5 \mathrm{~min}$, and the final equilibrium adsorbed concentration reaches $966.7 \mathrm{mg} \cdot \mathrm{g}^{-1}$ after $60 \mathrm{~min}$. The adsorption capacity of the as-prepared $\mathrm{Ni}$ powders is superior to that of the materials reported before [22-24]. Furthermore, we carried out FT-IR testing for the Ni particles before and after adsorption using infrared spectrometer to prove that CR have been absorbed into the sample. Figure 3(b) shows the curves of pure metal nickel and nickel after adsorption, from which a weak peak at 1058 of C$\mathrm{N}$ indicates the presence of CR. So they can be potentially 


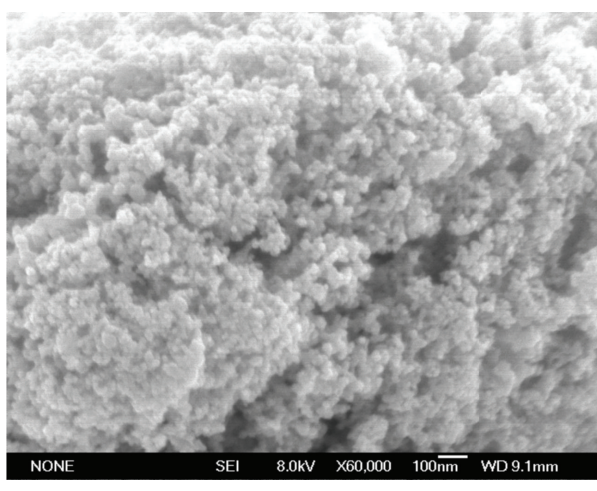

(a)

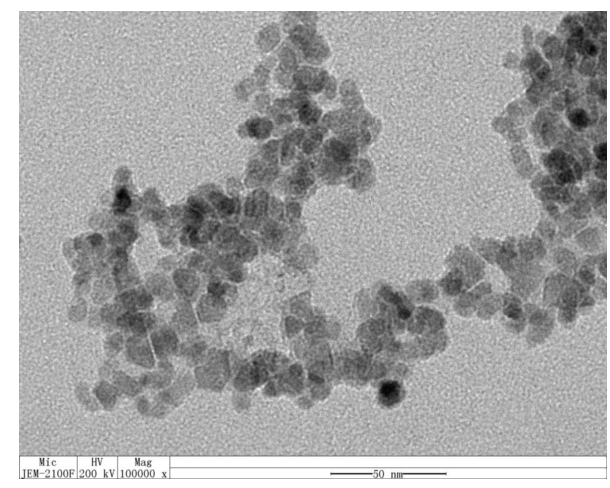

(b)

FIGURE 2: (a) SEM image and (b) TEM micrograph of Ni nanoparticles.

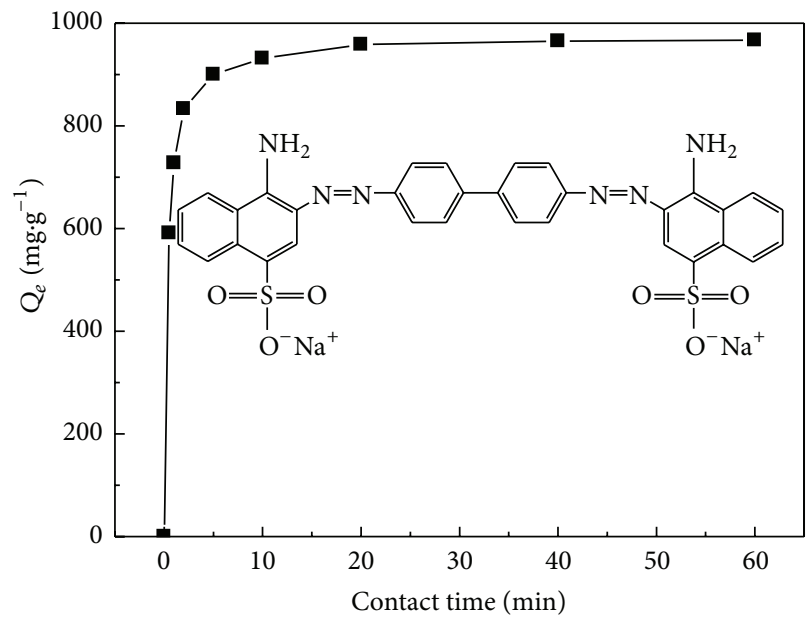

(a)

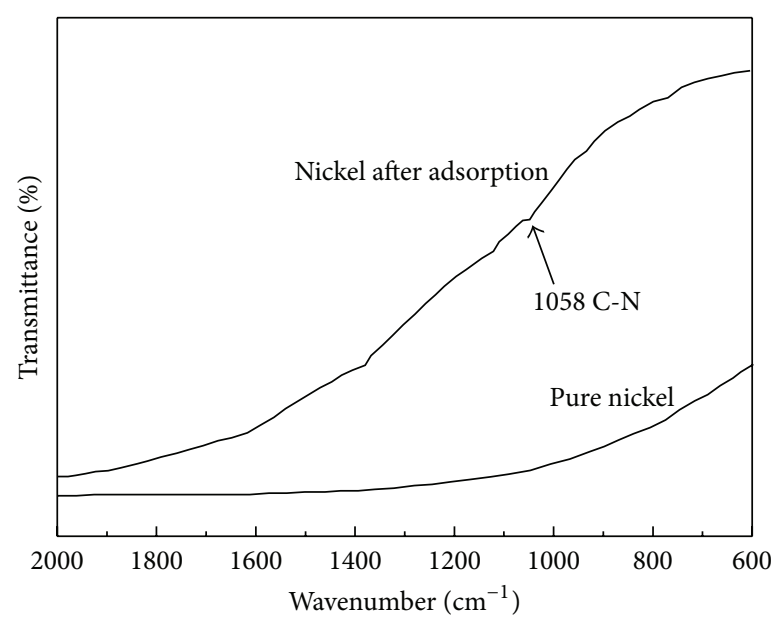

(b)

FIgURE 3: (a) Curve of adsorption capacities of Ni nanoparticles for CR and (b) FT-IR spectra of Ni nanoparticles before and after adsorption. Inset is the molecular structure of CR.

used as absorbent in the treatment of industrial wastewater containing $\mathrm{CR}$ within a short time.

The magnetic properties of the sample have been measured at room temperature (Figure 4). As shown in the magnetic hysteresis loop curve, the value of saturation magnetization (Ms) is $26 \mathrm{emu} \cdot \mathrm{g}^{-1}$; and the value of coercivity $(\mathrm{Hc})$ is $131 \mathrm{Oe}$. Compared to the bulk nickel material $(\mathrm{Ms}=$ $50 \mathrm{emu} \cdot \mathrm{g}^{-1}$ and $\mathrm{Hc}=100 \mathrm{Oe}$ ), the decreasing Ms and increasing Hc may be attributed to the incomplete crystallization and high surface/volume ratio due to the nanosized diameter of particles through quick chemical action at room temperature. Inset in Figure 4 represented the photograph of adsorption and magnetic separation behavior. A colorless solution was gained. More importantly, simple and rapid separation of CR-loaded magnetite adsorbent from treated water can be achieved via an external magnetic field. This indicates that the as-prepared sample belongs to ferromagnetic material and so can be easily separated from the dispersion system with the magnetic bar.

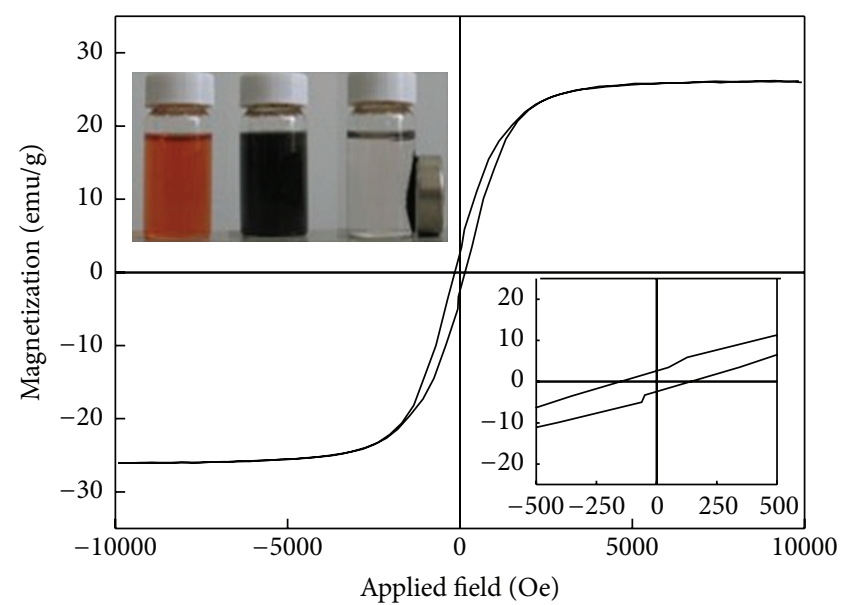

FIGURE 4: Magnetic hysteresis curve of Ni nanoparticles. Inset in turn is CR solution, mixing with the magnetic absorbents and separation of the adsorbent from solution with a magnet after adsorption, respectively. 


\section{Conclusions}

In summary, magnetic metallic nickel nanoparticles with the particle sizes in the range of 10 to $30 \mathrm{~nm}$ have been prepared by an easy $\mathrm{NaBH}_{4}$ reduction process at atmosphere temperature. The sample shows high adsorption capacity of $966.7 \mathrm{mg} \cdot \mathrm{g}^{-1}$ and fast adsorption rate of $83 \%$ within $5 \mathrm{~min}$ for the CR solution. Furthermore, the Ms value of the $\mathrm{Ni}$ nanoparticles is $26 \mathrm{emu} \cdot \mathrm{g}^{-1}$, which contribute to the removal of $\mathrm{Ni}$ with $\mathrm{CR}$. In conclusion, the as-prepared product can be used as a potential adsorbent in the process of industry wastewater especially containing CR.

\section{Conflict of Interests}

The authors declare that there is no conflict of interests regarding the publication of this paper.

\section{Acknowledgment}

This work was financially supported by the Social Development Projects of Jilin Province (20120406).

\section{References}

[1] G. Crini, "Non-conventional low-cost adsorbents for dye removal: a review, Bioresource Technology, vol. 97, no. 9, pp. 1061-1085, 2006.

[2] Y.-W. Jun, Y.-Y. Jung, and J. Cheon, "Architectural control of magnetic semiconductor nanocrystals," Journal of the American Chemical Society, vol. 124, no. 4, pp. 615-619, 2002.

[3] B. Kim, S. L. Tripp, and A. Wei, "Self-organization of large gold nanoparticle arrays," Journal of the American Chemical Society, vol. 123, no. 32, pp. 7955-7956, 2001.

[4] Y. H. Ni, X. W. Ge, Z. C. Zhang, and Q. Ye, "Fabrication and characterization of the plate-shaped $\gamma$ - $\mathrm{Fe}_{2} \mathrm{O}_{3}$ nanocrystals," Chemistry of Materials, vol. 14, no. 3, pp. 1048-1052, 2002.

[5] M. P. Pileni, "Nanocrystal self-assemblies: fabrication and collective properties," The Journal of Physical Chemistry B, vol. 105, no. 17, pp. 3358-3371, 2001.

[6] P.-Z. Li, A. Aijaz, and Q. Xu, "Highly dispersed surfactant-free nickel nanoparticles and their remarkable catalytic activity in the hydrolysis of ammonia borane for hydrogen generation," Angewandte Chemie, vol. 51, no. 27, pp. 6753-6756, 2012.

[7] N. Cordente, M. Respaud, F. Senocq, M.-J. Casanove, C. Amiens, and B. Chaudret, "Synthesis and magnetic properties of nickel nanorods," Nano Letters, vol. 1, no. 10, pp. 565-568, 2001.

[8] J. C. Bao, C. Y. Tie, Z. Xu, Q. F. Zhou, D. Shen, and Q. Ma, "Template synthesis of an array of nickel nanotubules and its magnetic behavior," Advanced Materials, vol. 13, no. 21, pp. 16311633, 2001.

[9] J. Bao, Y. Liang, Z. Xu, and L. Si, "Facile synthesis of hollow nickel submicrometer spheres," Advanced Materials, vol. 15, no. 21, pp. 1832-1835, 2003.

[10] H. L. Niu, Q. W. Chen, M. Ning, Y. S. Jia, and X. J. Wang, "Synthesis and one-dimensional self-assembly of acicular nickel nanocrystallites under magnetic fields," Journal of Physical Chemistry B, vol. 108, no. 13, pp. 3996-3999, 2004.

[11] X. M. Ni, Q. B. Zhao, D. Zhang, X. J. Zhang, and H. G. Zheng, "Novel hierarchical nanostructures of nickel: self-assembly of hexagonal nanoplatelets," Journal of Physical Chemistry C, vol. 111, no. 2, pp. 601-605, 2007.
[12] Ö. Metin, V. Mazumder, S. Özkar, and S. Sun, "Monodisperse nickel nanoparticles and their catalysis in hydrolytic dehydrogenation of ammonia borane," Journal of the American Chemical Society, vol. 132, no. 5, pp. 1468-1469, 2010.

[13] A. S. Manukyan, A. A. Mirzakhanyan, G. R. Badalyan et al., "Nickel nanoparticles in carbon structures prepared by solidphase pyrolysis of nickel-phthalocyanine," Journal of Nanoparticle Research, vol. 14, no. 7, article 982, 2012.

[14] J. Zhang and C. Q. Lan, "Nickel and cobalt nanoparticles produced by laser ablation of solids in organic solution," Materials Letters, vol. 62, no. 10-11, pp. 1521-1524, 2008.

[15] L. Y. Bai, F. L. Yuan, and Q. Tang, "Synthesis of nickel nanoparticles with uniform size via a modified hydrazine reduction route," Materials Letters, vol. 62, no. 16, pp. 2267-2270, 2008.

[16] G. Zhang, X. Zhao, and L. Zhao, "Preparation of single-crystalline nickel nanoflowers and their potential application in sewage treatment," Materials Letters, vol. 66, no. 1, pp. 267-269, 2012.

[17] C. Namasivayam and D. Kavitha, "Removal of Congo Red from water by adsorption onto activated carbon prepared from coir pith, an agricultural solid waste," Dyes and Pigments, vol. 54, no. 1, pp. 47-58, 2002.

[18] A. Afkhami and R. Moosavi, "Adsorptive removal of Congo red, a carcinogenic textile dye, from aqueous solutions by maghemite nanoparticles," Journal of Hazardous Materials, vol. 174, no. 1-3, pp. 398-403, 2010.

[19] L. X. Wang, J. C. Li, Y. Q. Wang, L. J. Zhao, and Q. Jiang, "Adsorption capability for Congo red on nanocrystalline $\mathrm{MFe}_{2} \mathrm{O}_{4}$ ( $\mathrm{M}=\mathrm{Mn}, \mathrm{Fe}, \mathrm{Co}, \mathrm{Ni})$ spinel ferrites," Chemical Engineering Journal, vol. 181-182, pp. 72-79, 2012.

[20] G. S. Zhang and L. J. Zhao, "Synthesis of nickel hierarchical structures and evaluation on their magnetic properties and Congo red removal ability," Dalton Transactions, vol. 42, no. 10, pp. 3660-3666, 2013.

[21] X. M. Liang and L. J. Zhao, "Room-temperature synthesis of airstable cobalt nanoparticles and their highly efficient adsorption ability for Congo red," RSC Advances, vol. 2, no. 13, pp. 54855487, 2012.

[22] S. Chatterjee, M. W. Lee, and S. H. Wooa, "Adsorption of congo red by chitosan hydrogel beads impregnated with carbon nanotubes," Bioresource Technology, vol. 101, no. 6, pp. 1800-1806, 2010.

[23] L. Wang and A.-Q. Wang, "Removal of Congo red from aqueous solution using a chitosan/organo-montmorillonite nanocomposite," Journal of Chemical Technology and Biotechnology, vol. 82, no. 8, pp. 711-720, 2007.

[24] S. Chatterjee, M. W. Lee, and S. H. Woo, "Influence of impregnation of chitosan beads with cetyl trimethyl ammonium bromide on their structure and adsorption of Congo red from aqueous solutions," Chemical Engineering Journal, vol. 155, no. 1-2, pp. 254-259, 2009. 

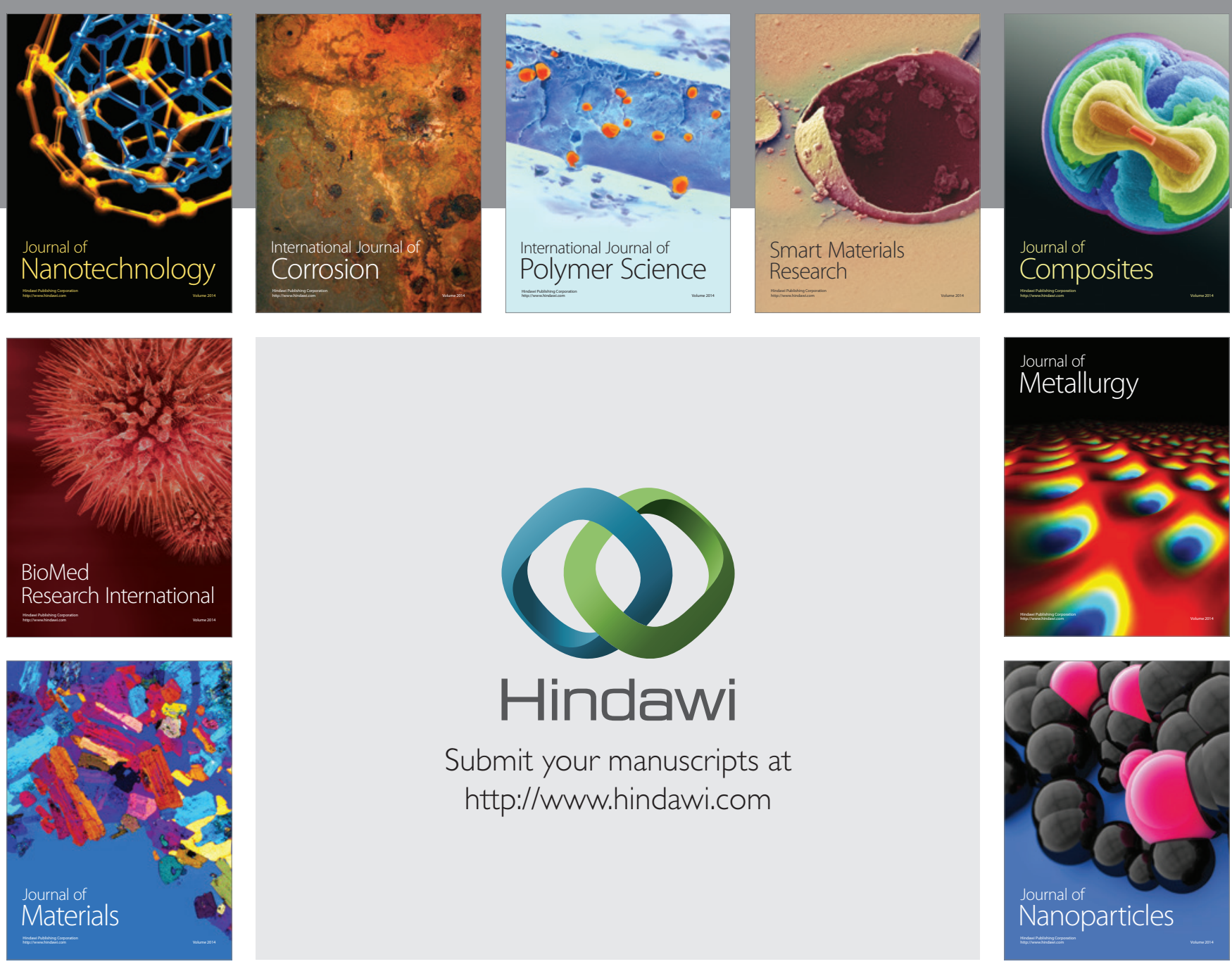

Submit your manuscripts at http://www.hindawi.com
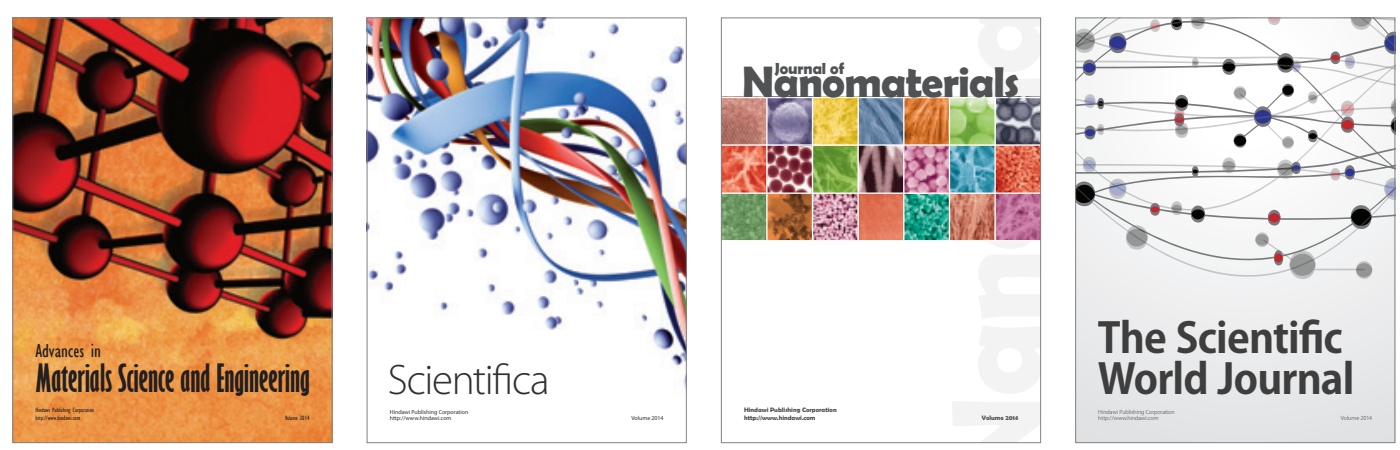

\section{The Scientific World Journal}
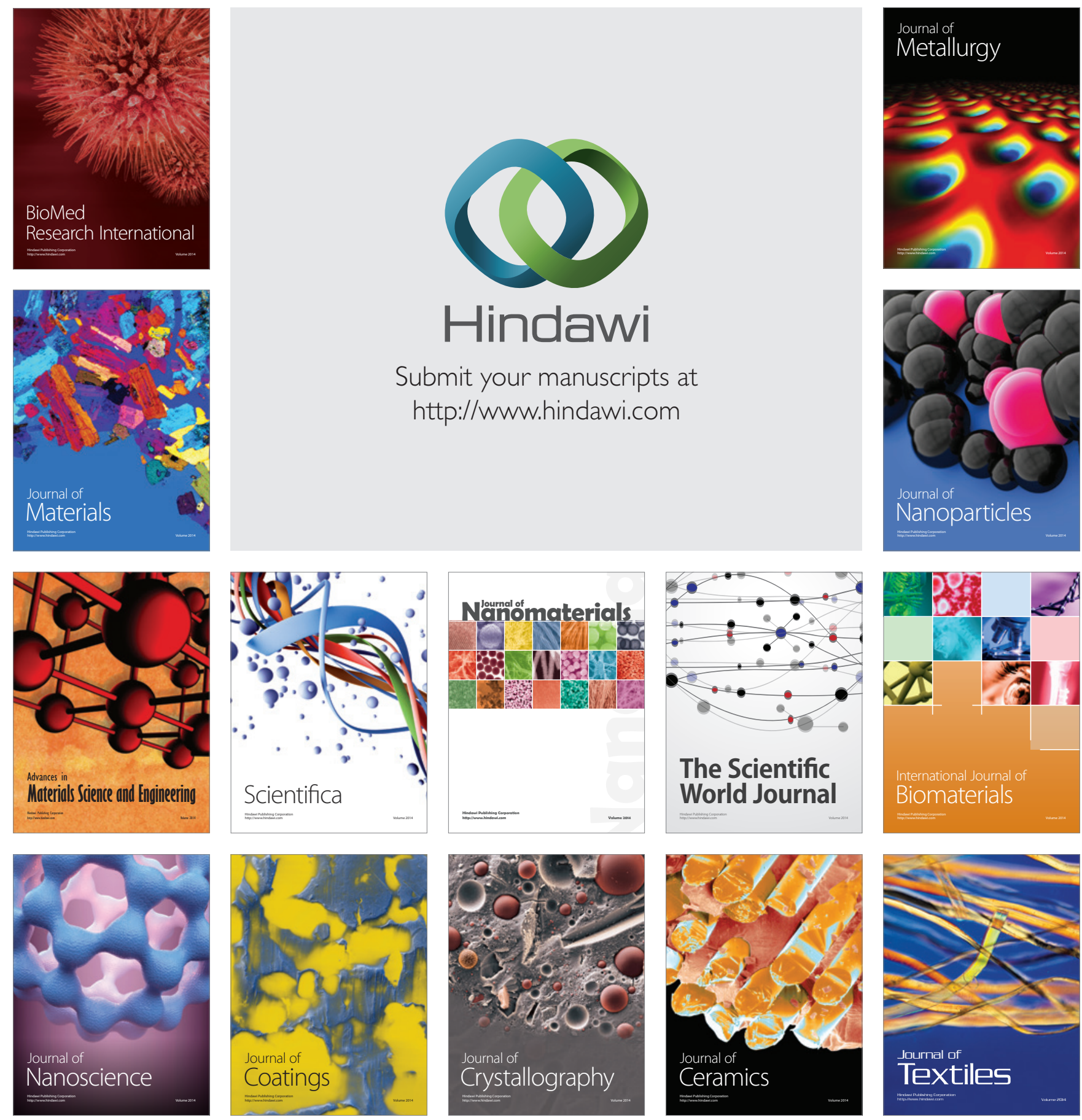